\title{
MOLECULAR ANALYSIS REVEALS UNIQUE MICROBIOME IN ILEAL POUCH DURING POUCHITIS COMPARED TO HEALTHY POUCHES IN ULCERATIVE COLITIS AND FAMILIAL ADENOMATOUS POLYPOSIS
}

\author{
A Thesis \\ Presented to \\ The Faculty of California Polytechnic State University, \\ San Luis Obispo \\ In Partial Fulfillment \\ of the Requirements for the Degree \\ Master of Science in Biological Sciences
}

by

Tiffany Wallingford Glavan June 2011 
(C) 2011

Tiffany Wallingford Glavan

ALL RIGHTS RESERVED 


\section{COMMITTEE MEMBERSHIP}

TITLE:

Molecular Analysis Reveals Unique Microbiome in Ileal

Pouch During Pouchitis Compared to Healthy Pouches in

Ulcerative Colitis and Familial Adenomatous Polyposis
AUTHOR:
Tiffany Wallingford Glavan
DATE SUBMITTED: June 2011

COMMITTEE CHAIR: Dr. Christopher Kitts, Ph.D., Associate Professor

COMMITTEE MEMBER: Dr. Candace Winstead, Ph.D., Assistant Professor

COMMITTEE MEMBER: Dr. Michael Black, Ph.D., Associate Professor 


\section{ABSTRACT}

Molecular Analysis Reveals Unique Microbiome in Ileal Pouch During Pouchitis Compared to Healthy Pouches in Ulcerative Colitis and Familial Adenomatous Polyposis

\section{Tiffany Wallingford Glavan}

In severe cases of ulcerative colitis (UC) unresponsive to current treatment options, patients require a complete proctocolectomy, or surgical removal of the colon. Ileal pouch anal anastomosis (IPAA) has become the preferred surgical technique for patients who require surgery, as this method restores rectal function. This procedure is also used to treat colorectal cancers such as adenocarcinoma and familial adenomatous polyposis (FAP). The surgery involves an abdominal colectomy with the construction of an ileal pouch created from folded tissue recovered from the ileal portion of the small intestine. Up to $50 \%$ of patients who require IPAA surgery experience an episode of pouchitis, a non-specific inflammation of the constructed ileal pouch with unknown etiology. Several hypotheses have been proposed regarding the pathogenesis of pouchitis. Current theories include bacterial overgrowth due to fecal stasis, microbial imbalance (dysbiosis), immune alteration, genetic susceptibility, metaplasia, ischemic complications of surgery, a recurrence of UC, or even a novel form of inflammatory bowel disease. The efficacy of antibiotics and probiotics in treating pouchitis and maintaining remission underscores the importance of gut microbiota in the development of this condition. In the study, we aimed to characterize the intestinal bacterial communities that inhabit IPAA pouches of both UC and FAP patients, in an effort to investigate the hypothesis that bacterial dysbiosis is involved in the pathogenesis of pouchitis. Mucosal biopsy and stool samples were analyzed from patients with UC and pouchitis (UCP), healthy UC controls (HUC) and healthy pouches with a background of FAP (FAP). Samples were examined through analysis of terminal restriction fragment length polymorphisms (TRF) and DNA sequencing. The data presented here demonstrate that a microbial imbalance exists in pouchitis, as bacterial communities in pouchitis differ significantly from healthy UC pouches and pouches constructed for FAP. Both methods identified potential groups of organisms that may play a role in the development of pouchitis, including decreases in protective Lactobacillus and Bacteroides and increases in mucin-degrading Clostridium and Akkermansia. A better understanding of the factors driving the pathogenesis of pouchitis will not only benefit patients with this disease, but also lead to a better understanding of the complex 
relationship that exists between the human host and the diverse community of organisms that inhabit the gastrointestinal tract.

Keywords:

Terminal Restriction Fragment Length Polymorphism

Ulcerative Colitis

Familial Adenomatous Polyposis

Pouchitis

Dysbiosis 


\section{ACKNOWLEDGEMENTS}

I would like to thank my advisor, Dr.Christopher Kitts, for his intellectual guidance and support, and for inspiring me to pursue a career in microbiology. Thank-you to Alice Hamrick and Anna Engelbrektson for their technical support and friendship. Thank-you to Dr.Garrett Zella for his persistence. Thank-you to the patients enrolled in this study for their participation. Thank-you to my committee for their patience. Thank-you to my family for their continued support and encouragement. 


\section{TABLE OF CONTENTS}

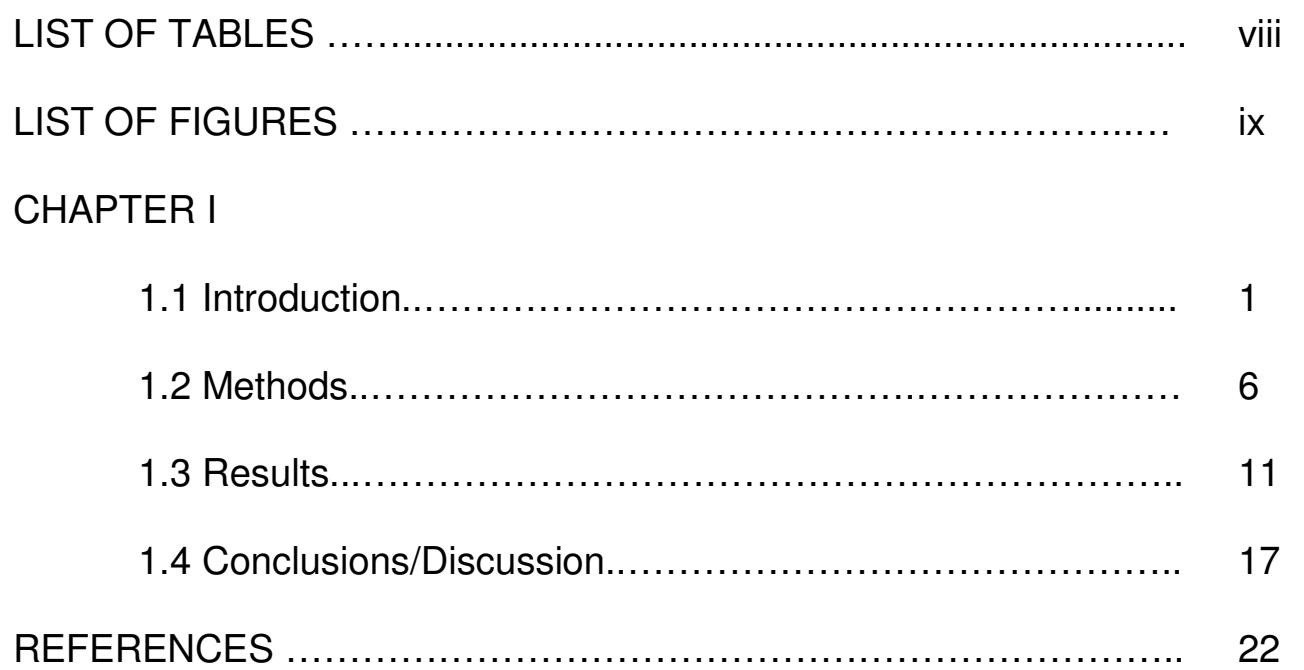




\section{LIST OF TABLES}

1. Patient Demographic and Disease Characteristics ................ 11

2. Comparison of DNA Sequencing Results by Phylum................ 15

3. Identifiable 16S rDNA Sequences in Fecal Samples of the UCP

Subgroup Compared to the FAP Subgroup............................. 


\section{LIST OF FIGURES}

Figure

1. Representative TRF Profiles.......................................... 12

2. Multidimensional scaling (MDS) analysis.............................. 14 


\subsection{INTRODUCTION}

Ulcerative colitis (UC) is a chronic but intermittent inflammatory bowel disease (IBD) characterized by continuous ulcers, or open sores, in the mucosal layer lining the large intestine [1]. This disease has a prevalence of 246 per 100,000 in the United States, equating to approximately 700,000 Americans suffering from symptoms including abdominal pain and cramping, bloody diarrhea, and fever [2]. The symptoms range in severity and can be localized to the rectum or involve the entire colon, but unlike Crohn's disease, UC is restricted to the large intestine [1]. The etiology of this condition is unknown, but studies indicate that both genetic and environmental factors are important. Hypotheses regarding the origin of UC include an autoimmune response to an antigen, a dysfunctional immune response to commensal organisms, or an infection with a pathogenic microbe [1].

Treatment for UC includes anti-inflammatory drugs and immunomodulators, which effectively suppress the disease in most cases [1]. Approximately $20-30 \%$ of patients, however, do not respond effectively to drug therapy and instead require surgical intervention [3]. Unlike Crohn's disease, ulcerative colitis can be cured, in some cases, by removal of the large intestines. The restorative proctocolectomy with ileal pouch anal anastomosis (IPAA) has become the preferred surgical procedure used to treat patients with severe UC [4]. This method involves an abdominal colectomy with the creation of a pouch that is connected to the anus. The pouch is constructed from loops of folded tissue recovered from the ileal portion of the small intestine. The alternative surgical procedure involves the connection of the small intestine directly through the abdominal wall. IPAA surgery is favored because the rectal muscular cuff and anal sphincter are left intact, eliminating the need for a collection bag.

Although IPAA surgery has functional advantages over previously used surgical techniques, a significant number of patients exhibit post-surgical complications. The 
most common is pouchitis, a nonspecific inflammatory condition that occurs in the reconstructed ileal pouch. Approximately one-half of all UC patients who receive IPAA surgery will develop pouchitis during their lifetime, a syndrome involving watery, frequent diarrhea accompanied by urgency, incontinence, abdominal cramping, and fever [5-6]. Although the majority of pouchitis patients respond to antibiotics, the condition will frequently return and becomes recurrent in approximately $10-15 \%$ of patients $[5,7]$.

Several hypotheses have been proposed regarding the pathogenesis of pouchitis. Current theories include the following: bacterial overgrowth due to fecal stasis, bacterial dysbiosis (imbalance), immune alteration, genetic susceptibility, metaplasia, ischemic complications of surgery, a recurrence of UC, or even a novel form of IBD [8]. Multiple lines of evidence point to a pivotal role for bacteria in pouchitis. First of all, inflammation of the mucosa has been shown to be localized to areas with the highest concentration of bacteria [9]. In addition, sonicated flora from pouchitis patients induces activation of ex vivo mononuclear cells while sonicates from healthy patients did not [10]. Furthermore, symptoms associated with pouchitis are often effectively treated with antibiotics [11-12]. Four week treatment of metronidazole with ciprofloxacin has been shown to be highly effective in patients with recurrent or refractory pouchitis [13]. Metronidazole accumulates in anaerobes and produces toxic free radicals, while ciprofloxacin is a broad spectrum antibiotic that inhibits DNA gyrase, halting bacterial replication. These two antibiotics have been shown to work best synergistically [7, 14]. Additional evidence supporting the involvement of intestinal microflora in pouchitis is the efficacy demonstrated by probiotic VSL\#3 in maintaining remission after recovering from an episode of pouchitis $[7,15]$. VSL\#3, marketed as "The Living Shield", is s mixture of eight different strains of bacteria, including Bifidobacteria, Lactobacillus, and Streptococcus. A once daily high dose administration of VSL\#3 has been shown to be effective in maintaining antibiotic introduced remission in patients experiencing 
recurrent pouchitis or pouchitis refractory to therapy [7]. Patients treated with VSL\#3 are shown to have lower levels of mucosal mRNA expression of proinflammatory cytokines IL-1 $\beta$, IL-8, and IFNy [16]. VSL\#3 has also demonstrated efficacy in treating ulcerative colitis [17]. Single-strain probiotic Lactobacillus rhamnosus GG has also been tested in cases of pouchitis, but effects were limited [18].

This clinical evidence has prompted much investigation into the relationship between pouchitis and intestinal bacteria. The results of these studies, however, are mixed, and no study has been able to identify a single pathogenic organism or toxin responsible for pouchitis. Multiple groups have used culture-based techniques that indicate a decrease in the ratio of anaerobic to aerobic bacteria in pouchitis [19-21]. Several studies cite an increase in sulfate reducing bacteria in the pouches of UC patients and implicate the production of hydrogen sulfide gas in the development of pouchitis [22-23]. Other studies have implicated increases in Clostridia or Fusobacter and decreases in Lactobacilli, Bifidobacteria or Streptococci [24-26]. Yet other studies indicate no difference in microbiological communities between inflamed and healthy pouches [22, 27-29].

Part of the reason for the inconsistent findings between studies is the high subjectivity in the classification of pouchitis. Prior to 1994, the criteria used in the diagnosis of pouchitis varied widely between clinicians. A classification system has since been developed in order to objectively quantify the condition of pouchitis, using a diagnostic scoring system known as the pouchitis disease activity index (PDAI) [30]. The PDAl score is determined using three principal components: symptoms (0-6pts), endoscopy (0-6pts), and histology (2-6pts). The clinical section of the PDAI rates symptoms such as stool frequency, rectal bleeding, fecal urgency or abdominal cramping, and fever. The level of inflammation is described during endoscopy through observation of factors such as edema, granularity, and ulceration. The histological 
section of the score considers levels of polymorphic nuclear leukocyte infiltration and ulcerations per low-power field. A combined score greater than seven qualifies as pouchitis. The use of the PDAI scoring system has led to more consistent classification of pouchitis patients.

Another probable reason for the discrepancies in findings between studies is the variation in the types of control samples used for comparison to pouchitis. It is difficult to compare ileal pouch flora from pouchitis patients directly to ileal flora of healthy patients, due to inherent challenges in collecting samples from the small intestines. The majority of studies compare inflamed UC pouches to healthy UC pouches or to ileostomy. The problem with this comparison is that it does not detect differences that may be due to the underlying condition of ulcerative colitis, since all samples have a background of the disease. In the study presented here, patients who receive IPAA surgery for UC are compared to patients who underwent the same surgery for familial adenomatous polyposis (FAP). FAP is a heritable condition in which hundreds to thousands of polyps form in the mucosal surface lining the intestine. If not treated, these polyps almost inevitably transform into malignancies, therefore surgery is employed in FAP cases to eliminate the risk of colon cancer. Patients who undergo IPAA surgery due to familial adenomatous polyposis (FAP) are ten times less likely to experience symptoms of pouchitis [31-32].

In addition to subjectivity in classification and variation in the types of samples compared, a third reason for inconstant findings within pouch microbiota studies is high variability in the experimental techniques (compounded by high intersubject variability). Available methodologies for studying gut microbiota have changed dramatically over the past twenty years. Original culture based techniques were problematic with gut samples, due to the high numbers of anaerobes and the difficulty in growing fastidious organisms or those with mutualistic dependence on other species. It is estimated that only about 
$20 \%$ of organisms can be cultured from the gut. Molecular techniques for community fingerprinting are now more commonly used and are typically based on variability within conserved regions of the $16 \mathrm{~S}$ ribosomal RNA gene. The techniques are similar in that all start with PCR using primers for sequences in this gene that are conserved in all prokaryotes. The methods differ in how the fragments are separated. Density Gradient Gel Electrophoresis (DGGE) uses chemical gradients of urea and formamide to separate fragments and temperature gradient gel electrophoresis (TGGE) is based on sequence dependent differences in the temperature at which double stranded DNA melts. In Terminal Restriction Fragment Length Polymorphism (TRFLP or TRF) one end of each PCR-amplified fragment is fluorescently labeled and the fragments are digested with restriction enzymes and separated using capillary gel electrophoresis on an automated sequence analyzer. TRF data have the advantage of being simply and rapidly produced, providing more information than DGGE or TGGE. Data are easily converted into a form that can be analyzed using a variety of statistical approaches and databases are available for comparison. Ideally, TRF is used for monitoring bacterial community structure over time. The resolution of TRF is, however, limited, as fragment sizes overlap in most cases, making absolute identification impossible. As more restriction enzymes are used, however, more information is available to differentiate species of bacteria. In addition to analysis using TRF profiles, PCR amplicons derived from this study were also sent for DNA sequencing. Sequences of $16 \mathrm{~S}$ ribosomal RNA genes provide more power to precisely identify the community composition in inflamed pouches versus healthy controls.

Both colonic biopsies and stool samples were obtained from IPAA patients enrolled in this study. The concurrent analysis of both mucosal and luminal samples is a strength of this study compared to earlier studies that generally assessed only stool samples. Analysis of mucosa is important since it has been shown that microorganisms 
interacting with the mucosa are significantly different than those found in fecal samples, and the interaction between the organisms is thought to be integral to the development of pouchitis [25, 33]. Only two other pouchitis studies compared mucosal and fecal flora $[20,25]$.

The aim of this study was to characterize the intestinal bacteria that inhabit IPAA pouches of both UC and FAP patients to investigate the hypothesis that an imbalance in bacterial populations is involved in the pathogenesis of pouchitis. Mucosa and stool samples were analyzed from patients with UC and pouchitis, healthy UC controls and FAP controls. Samples were examined using both TRF and DNA sequencing. The data presented here demonstrate that bacterial dysbiosis exists in pouchitis and the bacterial communities in pouchitis differ significantly from healthy UC pouches as well as from pouches constructed for FAP. Both methods identified potential groups of organisms that may play a role in the development of pouchitis. A better understanding of the factors driving the pathogenesis of pouchitis will not only benefit patients with this disease, but also lead to a better understanding of the complex relationships that exist between the human host and the diverse community of organisms that inhabit the gastrointestinal tract.

\subsection{METHODS}

Inclusion Criteria

Patients who have received care for a restorative proctocolectomy with IPAA at Massachusetts General Hospital were invited to participate in this study. Patients were enrolled in this study only if IPAA surgery was performed more than three months prior to enrollment. Patients were divided into three cohorts: those with UC and pouchitis (UCP); those with UC but no pouchitis (healthy UC pouch, HUC); and those with a history of FAP without pouchitis (healthy FAP pouch, FAP). Subjects enrolled as case subjects were those experiencing symptoms of at least three episodes of pouchitis in the 
past year. Pouch inflammation was documented by histological and endoscopic investigation and a PDAI score was assigned. PDAI scores greater than seven qualified the patient into the pouchitis (UCP) group. HUC control subjects included patients who had never experienced an episode of pouchitis or who had received a PDAI score less than seven.

\section{Exclusion Criteria}

Subjects taking probiotics, non-steroidal anti-inflammatory drugs, and immunosuppressants were excluded from the study, as well as any subject who had received topical immunosuppressant rectal therapy in the previous two weeks. Subjects chronically taking antibiotics were allowed in the study if the antibiotics were administered more than three months prior to sample collection and if the patient's symptoms persisted with antibiotic use. Subjects with any clinical evidence of Crohn's disease or any other immunological or hematological illness deemed significant to this study were excluded. Nineteen test subjects ultimately enrolled in the study and provided informed consent. The Massachusetts General Hospital and California Polytechnic State University human subject committees each approved the protocol and written consent was obtained from each individual.

Specimen collection

The pouch of each patient was examined to determine PDAI score. Each enrolled patient underwent a flexible sigmoidscopy performed by one of the study physicians. Patients received a bowel preparation one day in advance with phosphosoda. Both stool and mucosal biopsy specimens were obtained from each test subject. One $\mathrm{ml}$ stool samples were suctioned into three vials containing $10 \%$ glycerol $/ 90 \%$ sterile water and were immediately placed in dry ice. Biopsies were removed from the most inflamed area within the pouch, at least four centimeters from the anus. The biopsies were washed in $500 \mathrm{~mL}$ of saline and placed into a vial containing $10 \%$ 
glycerol $/ 90 \%$ sterile water were immediately placed in dry ice. Both types of samples were frozen at $-80^{\circ} \mathrm{C}$ within 30 minutes.

\section{DNA extractions}

DNA was isolated using MoBio's Power Soil DNA extraction kit, according to the manufacturer's protocol (MoBio Laboratories, Carlsbad, CA). Triplicate $0.1 \mathrm{~g}$ samples were used for the extraction of stool samples, while the entire biopsy sample was used for the isolation of DNA from tissue samples. The $16 \mathrm{~S}$ subunit bacterial ribosomal RNA gene was amplified from both isolates using the forward primer 8DF (5'-AGA GTT TGT TCM TGG CTC AG-3') and the reverse primer 536-K2R (5'-GTA TTA CCG CGG CTG CTG G-3'). The forward primer was fluorescently labeled with a phosphamide dye. $50 \mu \mathrm{l}$ reactions were assembled using $1 \mu \mathrm{l}$ of undiluted DNA, $5 \mu \mathrm{l}$ of $10 \mathrm{X}$ buffer, $3 \mu \mathrm{l}$ of $10 \mathrm{mM}$ dNTPs, $2 \mu$ of $20 \mathrm{mg} \mathrm{mL}^{-1} \mathrm{BSA}, 7 \mu \mathrm{l}$ of $25 \mathrm{mM} \mathrm{MgCl}_{2}, 1 \mu \mathrm{l}$ of each primer, and $0.3 \mu \mathrm{l}$ of 5 $U^{-1}$ TaqGold (Applied Biosystems, Foster City, CA). Reaction temperatures and times were as follows: $95^{\circ} \mathrm{C}$ for $10 \mathrm{~min}$ followed by 30 cycles of $95^{\circ} \mathrm{C}$ for $1 \mathrm{~min}, 60^{\circ} \mathrm{C}$ for $1 \mathrm{~min}$, and $72^{\circ} \mathrm{C}$ for $2 \mathrm{~min}$, followed by a final extension of $72^{\circ} \mathrm{C}$ for $10 \mathrm{~min}$. The quality of the extractions and PCR reactions were confirmed using gel electrophoresis. PCR triplicates were combined during a PCR cleanup performed using MoBio's PCR Cleanup Kit following the manufacturer's protocol (MoBio Laboratories, Carlsbad, CA). PCR products were quantified using a FLX800 microplate fluorescence reader tuned to the labeling dye (Bio-Tek Instruments; Winooski,VT).

\section{Digestion and Resolution of TRF patterns}

Enzyme digests were independently performed using cleaned-up PCR product and the restriction endonucleases Haell, Hpall, and Alul (New England Biolabs; Beverly, MA). Each $40 \mu$ digest included 75 ng DNA, $1 \mathrm{U}$ enzyme, and $4 \mu$ l buffer. Samples were digested for $4 \mathrm{~h}$ at $37^{\circ} \mathrm{C}$ and inactivated for $20 \mathrm{~min}$ at either $65^{\circ} \mathrm{C}$ (Hpall and Alul) or $80^{\circ} \mathrm{C}$ (HaellI). Resulting fragments were ethanol precipitated and 
resuspended in $20 \mu \mathrm{l}$ formamide and $0.25 \mu \mathrm{l}$ CEQ 600 base pair standard. Terminal restriction fragments were separated using capillary gel electrophoresis and profiles were obtained using a Beckman Coulter CEQ8000X DNA analysis system.

Terminal restriction fragment lengths (in nucleotides) and relative peak areas (relative abundance) were exported from the CEQ8000 into Excel (Microsoft, Seattle, WA). The area under each peak was normalized for loading and expressed in parts per million to standardize the data for comparison. Peaks with an area less than 10,000 ppm $(<1.0 \%$ of the total) were excluded from the analysis to reduce excess noise. Data from three independent restriction digests were performed to provide better resolution (reduce incidence of distinct sequences with equivalent TRF lengths) and thus help tentatively identify groups when performing database comparisons between fragment lengths and in silico restriction enzyme digests of 16S rRNA gene sequences in GenBank (NCBI, Bethesda, MD).

Data analysis

TRF data sets were transformed by taking the square root of the area under each peak to de-emphasize large TRF peaks while still accounting for relative abundance. Transformed data were compared using Bray-Curtis similarity, multidimensional scaling, and analysis of similarity (Primer, London, UK; Microsoft Excel, Seattle, WA). TRF fragments were subjected to ANOSIM analysis to determine the TRF fragments that differ most between groups. These fragments were compared to available GenBank sequences to tentatively identify potential bacterial populations associated with bacterial dysbiosis. The database for TRF matching was created by obtaining all 16S rRNA gene sequences from GenBank. These sequences were processed using in silico PCR and restriction enzyme digests. Observed TRF peaks were compared to the predicted lengths based on the database, allowing for a difference of one base pair between the lengths of observed and predicted fragments. 


\section{Statistics}

Transformed data were compared between groups using Bray-Curtis similarity, multidimensional scaling, and analysis of similarity (ANOSIM) (Primer E, Plymouth, UK). Bray-Curtis was used to determine overall level of similarity between groups. ANOSIM was used to identify the factors that made the groups different. ANOVA was used to compare relative abundances of specific TRF elements between groups.

\section{S Ribosomal DNA-based DNA Sequencing}

Four fecal samples from each of the UCP and FAP groups were selected and pooled for sequencing analysis. Samples were chosen based on greatest intersubject similarity, using Bray-Curtis similarity indices. Extracts from selected samples were subjected to PCR with the same primers used in TRF, without the fluorescent label. Resulting fragments were pooled and sent to the Broad Institute in Cambridge Massachusetts for sequencing.

Samples were cloned into pCR2.1-TOPO vectors and sequenced on an ABI3730 DNA sequencer. Resulting sequences were trimmed using LUCY, a tool that examines raw DNA sequence data for quality assurance [34]. Read pairs from each clone were assembled using an alignment-assisted assembly method implemented at the Broad Institute. 16S rRNA gene sequences obtained from Greengenes provided the alignment template used as a reference sequence for the read pairs [35]. The reference sequence with the greatest number of k-mers matching with the aggregate k-mer set of both forward and reverse reads was selected. The forward and reverse reads were aligned to the core reference sequence using basic local alignment search tool (NCBI, Bethesda, MD) [36]. Aligned forward and reverse reads were then assembled based on base quality scores. High quality reads longer than 1100 nucleotides were used for classification. Sequences were classified according to comparisons with the Ribosomal Database Project sequence collection [37-38]. 


\subsection{RESULTS}

\section{Patient Characteristics}

Nineteen patients were enrolled in the study. The samples were divided into groups based on the reason for IPAA surgery (ulcerative colitis vs. familial adenomatous polyposis) and the PDAI score (healthy pouch vs. pouchitis) (Table 1). Twelve patients received surgery for ulcerative colitis, and of these 9 had pouchitis (UCP) and 3 had healthy pouches. Seven patients were enrolled that received IPAA surgery for familial adenomatous polyposis. There were no significant differences between patient demographics (age and gender) or disease characteristics (duration of ulcerative colitis and pouch). PDAI scores were significantly higher in the pouchitis group, and each biopsy in this group were shown to display histologic evidence of active pouchitis as part of the PDAI score (defined by presence of polymorphonuclear infiltrates and ulcerations). Several patients enrolled in the UCP group were receiving chronic antibiotic therapy. Two patients were currently taking antibiotics, one for eight years and one for one year, while still having episodes of pouchitis. Three other patients had received short courses of antibiotics during the past year, but not during the three month time span prior to enrolling in the study.

\begin{tabular}{lcccc}
\hline \multicolumn{4}{l}{ TABLE 1. Patient Demographic and Disease Characteristics } \\
\hline UCP $(n=9)$ & FAP $(n=7)$ & HUC $(n=3)$ & $P$-value \\
\hline Mean age in years (range) & $32.2(22-61)$ & $45.5(29-55)$ & $39.3(26-55)$ & 0.15 \\
Gender & $\mathrm{M}=2(22 \%)$ & $\mathrm{M}=4(57 \%)$ & $\mathrm{M}=1(33 \%)$ & 0.48 \\
& $\mathrm{~F}=7(78 \%)$ & $\mathrm{F}=3(43 \%)$ & $\mathrm{F}=2(67 \%)$ & 0.51 \\
Mean duration of pouch in years (range) & $5.9(1.5-13)$ & $8.7(1.4-21)$ & $12.3(2-20)$ & 0.31 \\
Mean duration of UC in years (range) & $8.8(2.5-14)$ & $\mathrm{n} / \mathrm{a}$ & $14.7(3-25)$ & $<0.001 *$ \\
Mean PDAI score (range) & $8.8(7-12)$ & 0 & $1(1)$ & \\
*Statistically significant. UCP, UC pouchitis; FAP, familial adenomatous polyposis; HUC, healthy UC. & & \\
\hline
\end{tabular}




\section{Terminal Restriction Fragment Analysis}

The total number of peaks provides a measure of overall community diversity. There were significant differences in the total number of TRF peaks in the mucosal biospsies compared to stool (average total peaks, mucosa $=64.8$, feces $=41.3, p<0.001$ ). (Figure 1). Fecal and mucosal microbial fingerprints were significantly more similar within samples from a single subject compared to between subjects $(p=0.027)$. Compared to stool samples, mucosal samples taken as a whole revealed significantly greater intersubject percentage similarity $(p<0.001)$. The total number of fecal TRF peaks, $a$ marker of overall diversity, was lower in pouchitis compared to stool from healthy subjects without proctocolectomy (42.3 in UCP, 64.0 in normal stool, $p<0.001)$. TRFs used for this analysis were collected from healthy seniors at California Polytechnic State University as part of an independent study. However, HUC and FAP groups also showed significantly lower microbial diversity in the stool samples compared to healthy subjects without proctocolectomy $(\mathrm{HUC}=35.3, \mathrm{p}<0.05 ; \mathrm{FAP}=41.4, \mathrm{p}<0.005)$.

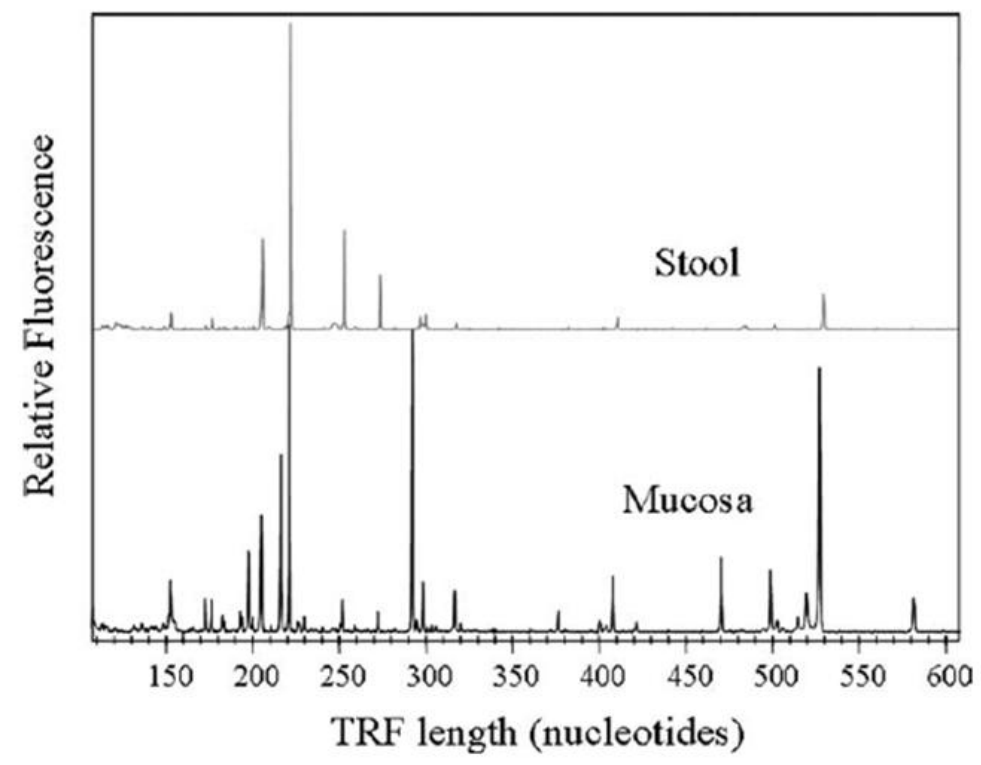

Figure 1. Representative TRF profiles. These two patterns were generated from the stool and mucosa of a single patient. The mucosa had a higher degree of community diversity, based on the total number of peaks. 
Overall, there was a higher degree of similarity within TRF profiles from the FAP group $(37.78 \%)$ compared to the UCP group, where microbial profiles were more variable overall $(20.11 \%)$. TRF profiles were significantly different between UCP and FAP groups when evaluating mucosal and stool separately, or when combined $(p<0.05$, Figure 2). Profiles were also distinct between UC patients with and without pouchitis $(p<0.05)$. No significant differences were observed in patients taking antibiotic therapy.

The analysis of similarity (ANOSIM) analysis from the three different restriction digests were compared to find sets of peaks that were most different between groups in each digest. This analysis revealed a set of TRFs matching Lactobacillus and Streptococcus (Hae 264-5, Hpa 97-9, Alu 76,532) were present at higher relative abundance in both mucosal and fecal samples from FAP patients compared to UCP (ratio 5:1 in mucosa, 3:1 in stool). A second set of TRF peaks (Hae 272-4, Hpa 222-3, Alu 440) matching Clostridium, Eubacterium and Roseburia genera were present at five time the relative abundance in stool from UCP patients compared to FAP patients. Fecal samples from HUC pouches also had fewer peaks matching Clostridium, Eubacterium and Roseburia compared to UCP (ratio 1:15). Profiles from healthy UC pouches had fewer Escherichia, Streptococcus, and various sulfur-oxidizing bacteria (Hpa 496, Hae 205, Alu 74) at a ratio of 1:2 compared to UCP. Mucosal samples from healthy UC pouches also showed less Escherichia, Streptococcus, and various sulfur-oxidizing bacteria compared to UCP (ratio 1:2). There were no groups that were increased in healthy UC pouches that were statistically significant. 


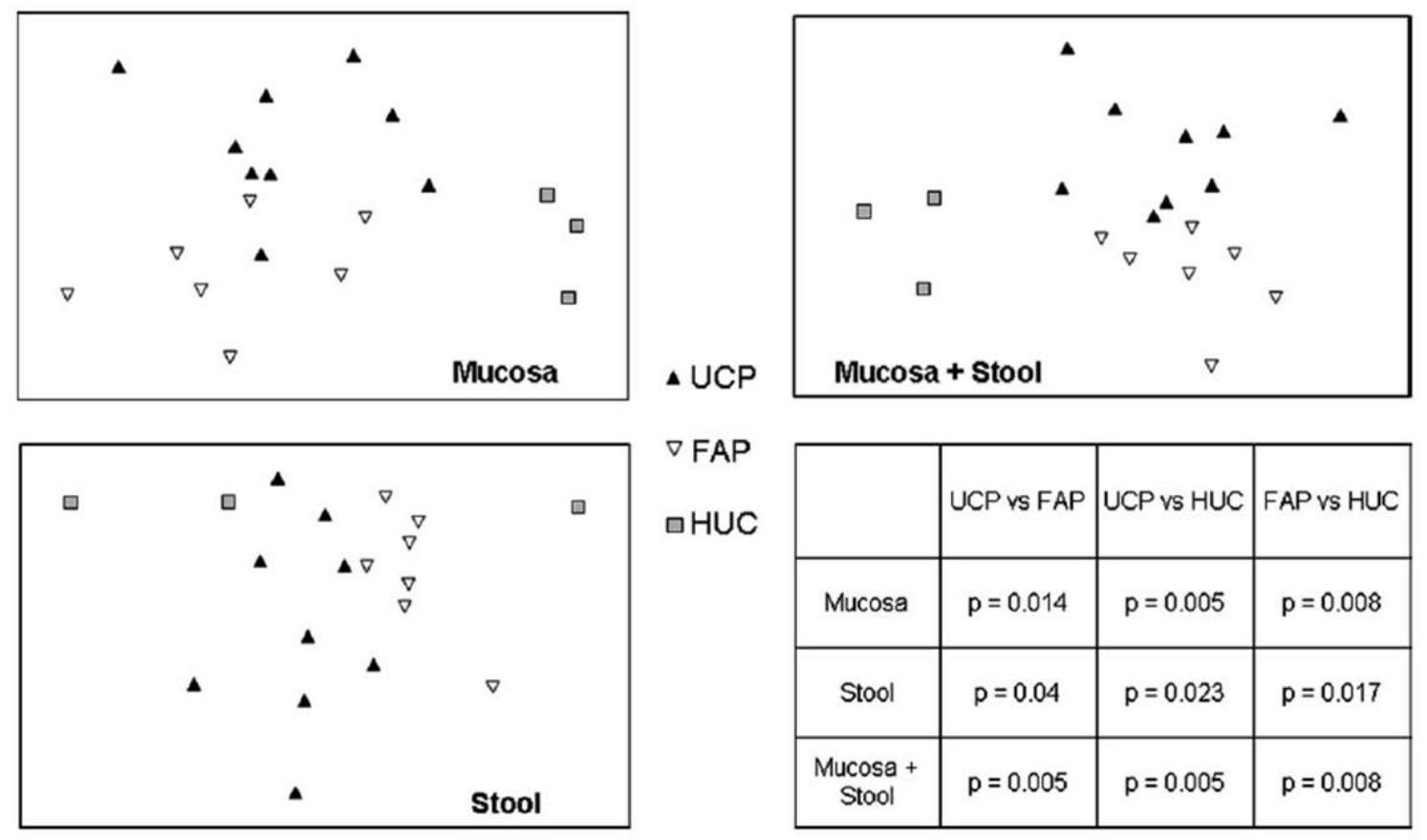

Figure 2. Multi-dimensional scaling (MDS) analysis. This analysis is used to compare TRF profiles between groups. Each symbol represents the profile of one subject and the distance between symbols is representative of overall similarity. Significant differences in microbial communities were observed between each experimental group.

\section{DNA Sequencing}

Pooled fragments from the UCP and FAP group were sent for cloning and sequencing at the Broad Institute. For each of the samples, 2304 clones were processed in total. In the UCP pooled sample, 712 sequences were identified at the genus level, and for the FAP samples, 1015 were identified. The groups were first compared at the phylum level. These data showed increased Firmicutes and Verrucomicrobia in the UCP group and more Bacteroidetes in the FAP group $(p<0.001)$ (Table 2). Sequences between groups were also compared at the genus level. Table 3 shows the classification of the sequences that were different between experimental groups. Like the TRF data, the sequencing data revealed more Clostridia, namely Roseburia in the UCP group and more Escherichia in the FAP group. 
TABLE 2. Comparison of DNA Sequencing Results by Phylum

\begin{tabular}{lccc}
\hline & \multicolumn{3}{c}{$\begin{array}{c}\text { Percent of Identifiable DNA Clones } \\
\text { (Absolute Number) }\end{array}$} \\
\cline { 2 - 4 } & UCP & FAP & $P$-value \\
\hline Bacteroidetes & $20 \%(144)$ & $71 \%(716)$ & $<0.001$ \\
Firmicutes & $52 \%(379)$ & $19 \%(216)$ & $<0.001$ \\
Fusobacteria & $1 \%(4)$ & $<1 \%(4)$ & NS \\
Proteobacteria & $5 \%(30)$ & $5 \%(44)$ & NS \\
Verrucomicrobia & $22 \%(155)$ & $3 \%(35)$ & $<0.001$ \\
Actinobacteria & $<1 \%(0)$ & $<1 \%(1)$ & NS \\
\hline NS, not significant; UCP, UC pouchitis; & FAP, familial & adenomatous \\
polyposis. & & & \\
\hline
\end{tabular}




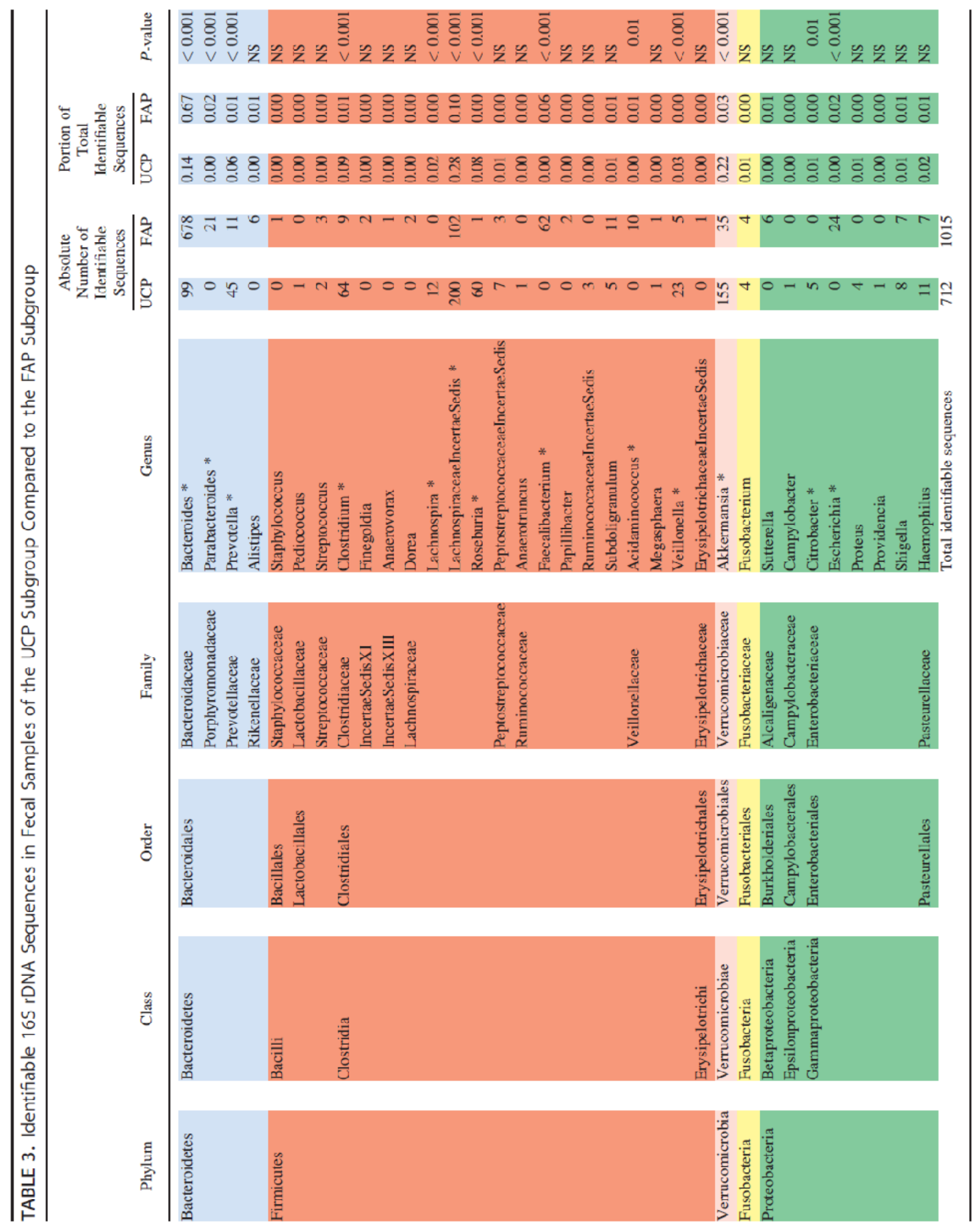




\subsection{Conclusions/Discussion}

The precise role of intestinal bacteria in the pathogenesis of pouchitis remains unclear. This investigation, however, has revealed the existence of distinct pouch environments in patients who have pouchitis and those with healthy pouches in UC and FAP, using TRF and DNA sequencing techniques based on sequence variability in the gene for $16 \mathrm{~S}$ rRNA. The pouch microbial environment, including both mucosal and luminal communities, is significantly altered in patients with UC-associated pouchitis compared to patients who received IPAA surgery in the setting of FAP. These findings demonstrate that bacterial dysbiosis exists in pouchitis and thus underscore the importance of the relationship between microbiota colonizing the gastrointestinal tract and the health of the host. This study was limited by the small number of healthy ulcerative colitis patients enrolled and the necessity of pooling fragments prior to DNA sequencing.

Multiple studies have indicated reductions in community diversity in pouchitis [27, 29], yet other studies demonstrate no change in diversity [39]. In TRF, diversity is represented by total number of peaks. The data collected here indicates that there is less community diversity in stool samples after IPAA surgery, indicated by fewer overall peaks in stool samples from all enrolled patients compared to stool from healthy subjects with intact colons. The finding is expected based on the fact that the colon supports a much greater bacterial load [40]. Between different experimental groups, however, there were not significant differences in total numbers of peaks between each of the three groups. The profiles of mucosal biopsies was shown to harbor greater numbers of total peaks than the fecal samples, providing supporting evidence for the idea that the bacteria found in stool comprise a subset of bacteria found in at the mucosal surface [33] (Figure 2). 
The bacterial community profiles were shown to be significantly different between all three experimental groups. This finding suggests that pouchitis is related to underlying factors in ulcerative colitis, since the communities found in healthy UC pouches was different from healthy FAP pouches. Although symptoms of UC are localized to the large intestine, underlying genetic and immune alterations (i.e. NOD2 polymorphisms or increased cytokine production) could lead to a similar pathology in the small intestines post-surgery. This finding is supported by clinical evidence that UC pouches are known to be 10X more susceptible to pouchitis than FAP pouches [32].

Both TRF and sequencing data indicate decreased abundance of protective Lactobacillus and Bacteroides in diseased pouches. Both species have welldemonstrated protective effects for the host. Lactobacillus is a known probiotic organism that protects the gut through competitive inhibition of other colonizing microbes. Certain species of Lactobacillus have been shown to reduce inflammation in the mouse model of inflammatory bowel syndrome, likely by modulating cytokine function. Lactobacillus have been shown to increase occludin and claudin production, upregulating tight junction formation and helping to maintain epithelial barrier function in the host. Several other studies have indicated decreased Lactobacillus in pouchitis [24, 26]. Bacteroidetes, specifically genus Bacteroides, typically comprise a large portion of the gastrointestinal tract. These mutualistic bacteria also provide competitive inhibition of pathogenic species, likely gaining a competitive advantage through the ability to process complex polysaccharides. Studies investigating the effects of probiotics on microbial flora report increases in Bacteroides after probiotic therapy [41], and this effect may help explain the efficacy demonstrated by probiotic therapy in pouchitis. In general, the importance of protective species is underscored by the efficacy of probiotic species in maintaining remission in pouchitis. 
Both TRF and sequencing data indicate increased abundance of Phylum Firmicutes in diseased pouches, including genus Clostridium and Roseburia. Clostridia are resistant spore-forming anaerobes and multiple members of this class are known human pathogens. Clostridium difficile is a common opportunistic pathogen that is known to increase in abundance after antibiotic treatment. C.difficile infections are common in pouchitis, with or without antibiotic treatment [42]. Several species of Clostridium are mucolytic and capable of breaking down the protective mucus layer in the host's intestinal lumen, potentially leaving the underlying epithelium more prone to invasion. Mucins provide an important energy source for bacteria as well as important barrier for the human host. In a healthy host, there exists a balance between the production of mucus by goblet cells and the breakdown of mucins by resident bacteria. An overabundance of Clostridium could disrupt this balance, due to enhanced glycosidase activity. Several other studies have demonstrated increases in Clostridia in pouchitis, including Clostridium perfringens [11, 24]. Members of genus Roseburia were also increased in diseased pouches. Roseburia are common flagellated butyrate producing bacteria. Bacterial flagellin has been shown to be a dominant antigen in Crohn's disease and, furthermore, serum IgG from patients with CD and mice with colitis both reacted specifically to flagellin from Roseburia [43]. Another group that was shown to be increased in diseased pouches was Akkermansia in Phylum Verrucomicrobia. This genus, like Clostridia, is known for mucin degradation. The loss of the protective mucus layer allows for direct contact between the intestinal epithelium and commensal organisms and may perpetuate inflammation.

These data, along with many previous studies, fail to pinpoint one specific group of bacteria associated with pouchitis, and therefore do not support the hypothesis that a single pathogenic organism leads to inflammation in pouchitis. Evidence in a shift of predominant microbial communities, however, was apparent. These findings provide 
support for the hypothesis that pouchitis involves alterations in the homeostasis of commensal organisms. Increased abundance of colitogenic bacterial species, such as Clostridia and Akkermansia identified in this study, may initiate inflammation by disrupting barrier function or destroying the mucosal surface of the host gastrointestinal tract.

It is, however, difficult to separate cause from consequence. Changes in microbial structure could initiate inflammation, but changes in immune function could drive changes in microbial communities. There is mounting evidence that links immune alterations to pouchitis, and it is hypothesized that a loss of tolerance to commensal organisms leads to inflammation. Ferrante et al retrospectively investigated serum antibody production and pattern recognition receptor polymorphisms in a large cohort of IPAA patients and found associations between pouchitis and outer-membrane porin antibodies and toll-like receptor 1 polymorphisms, implying immune dysfunction [44]. Another study demonstrated increased proliferation of immature plasma cells near ulcers and inflamed mucosa of pouchitis, suggesting an immune defect in B cell development [45]. Increases have been demonstrated in levels of activated mucosal CD4+ cells and multiple proinflammatory cytokines, including IFNy, IL-1ß, IL-6, IL-8, and TNFa [46-47]. High proinflammatory cytokine production promotes inflammation and can lead to intestinal epithelial destruction and crypt hyperplasia. Genetic susceptibility loci have also been identified that likely contribute to immune dysfunction. A recent study indicates that nucleotide oligomerization domain-2 (NOD2) mutations are found at higher frequencies in patients with severe pouchitis [48].

Although it is difficult to separate changes in community makeup and immune function in terms of cause and consequence, the importance of the relationship between commensal microorganisms and the human host are becoming increasingly clear. Hostmicrobe relationships in the gut are the focus of a rapidly growing field of research aimed 
at understanding how the two interact. Much advancement has been made in understanding the role of bacteria in human health. Germ-free mice were the first to demonstrate the critical role of bacteria in the development and maturation of the immune system, as mice raised in a sterile environment have altered development of both gut mucosal and peripheral immune systems, with a lack of expansion of CD4+ Tcells and a lack of lymphoid structure [49]. Stimulation by bacterial products is necessary and sufficient to initiate the maturation process [50].

In addition to being instrumental in the initial development of the immune system, bacteria also help maintain the health of the adult host. Some species of bacteria actively stimulate the host to produce anti-microbial peptides and proteins involved in tight junction formation, contributing to the maintenance of host epithelial integrity [40]. It is well established that bacteria help us digest complex polysaccharides, but only recently has it been appreciated that bacteria play a role in how we extract nutrients from food and in the uptake of lipids and dietary fiber. The composition of the gut microflora has recently been implicated in metabolic disorders such as obesity and diabetes [40]. As more and more data are compiled describing the composition of human intestinal microflora, enterotypes, or robust clusters of certain species, are emerging. Certain species drive the different enterotypes, based more on microbial functions (i.e. metabolism) than host factors (i.e. age or gender) [51]. It is hypothesized that persons with different microbial enterotypes respond differently to diet and drug intake.

Collectively, research on host-microbe interactions in the gut has demonstrated that there is a complex interplay in mucosal immunology between three systems that determine gastrointestinal health: host genetics, bacterial community structure, and immune function. Each system can influence the other systems and the wrong combinations of aberrant factors lead to mucosal pathogenesis. The pathogenesis of pouchitis, like gut health in general, is likely to be multi-factorial, involving overlapping 
factors and creating a spectrum of disorders rather than a single disease. Much work is left to be done to uncover the complex host-microbe relationships and interactions between microbes and the host. Future studies should aim to concurrently study microbiota while also investigating immune function through cytokine and antibody production, or attempt to link pouchitis with genetic polymorphisms in large patient cohorts. Knowledge gained from future research efforts in this area will have wide applications to improving human health and well-being, not only including patients suffering from pouchitis after IPAA surgery. The complexity and diversity of life in our intestines and the relationship between the host and resident bacterial community pose a significant challenge to researchers, but the knowledge gained from these studies has the potential to dramatically increase our understanding of human health.

\section{References}

1. Hendrickson, B.A., R. Gokhale, and J.H. Cho, Clinical aspects and pathophysiology of inflammatory bowel disease. Clin Microbiol Rev, 2002. 15(1): p. 79-94.

2. Loftus, E.V., Jr., Clinical epidemiology of inflammatory bowel disease: Incidence, prevalence, and environmental influences. Gastroenterology, 2004. 126(6): p. 1504-17.

3. Becker, J.M., Surgical therapy for ulcerative colitis and Crohn's disease. Gastroenterol Clin North Am, 1999. 28(2): p. 371-90, viii-ix.

4. Goldstein, N.S., W.W. Sanford, and J.H. Bodzin, Crohn's-like complications in patients with ulcerative colitis after total proctocolectomy and ileal pouch-anal anastomosis. Am J Surg Pathol, 1997. 21(11): p. 1343-53.

5. Lim, M., et al., Dysbiosis and pouchitis. Br J Surg, 2006. 93(11): p. 1325-34.

6. Mahadevan, U. and W.J. Sandborn, Diagnosis and management of pouchitis. Gastroenterology, 2003. 124(6): p. 1636-50.

7. Mimura, T., et al., Once daily high dose probiotic therapy (VSL\#3) for maintaining remission in recurrent or refractory pouchitis. Gut, 2004. 53(1): p. 108-14.

8. Cheifetz, A. and S. Itzkowitz, The diagnosis and treatment of pouchitis in inflammatory bowel disease. J Clin Gastroenterol, 2004. 38(5 Suppl): p. S44-50.

9. Sartor, R.B., Microbial influences in inflammatory bowel diseases. Gastroenterology, 2008. 134(2): p. 577-594. 
10. Bell, A.J., et al., Human lymphocyte stimulation with pouchitis flora is greater than with flora from a healthy pouch but is suppressed by metronidazole. Gut, 2004. 53(12): p. 1801-5.

11. Gosselink, M.P., et al., Eradication of pathogenic bacteria and restoration of normal pouch flora: comparison of metronidazole and ciprofloxacin in the treatment of pouchitis. Dis Colon Rectum, 2004. 47(9): p. 1519-25.

12. Gionchetti, P., et al., Antibiotic combination therapy in patients with chronic, treatment-resistant pouchitis. Aliment Pharmacol Ther, 1999. 13(6): p. 713-8.

13. Mimura, T., et al., Four-week open-label trial of metronidazole and ciprofloxacin for the treatment of recurrent or refractory pouchitis. Aliment Pharmacol Ther, 2002. 16(5): p. 909-17.

14. Gionchetti, P., et al., Diagnosis and treatment of pouchitis. Best Pract Res Clin Gastroenterol, 2003. 17(1): p. 75-87.

15. Campieri, M. and P. Gionchetti, Bacteria as the cause of ulcerative colitis. Gut, 2001. 48(1): p. 132-5.

16. Lammers, K.M., et al., Effect of probiotic strains on interleukin 8 production by HT29/19A cells. Am J Gastroenterol, 2002. 97(5): p. 1182-6.

17. Turcotte, J.F. and H.Q. Huynh, Treatment with the probiotic VSL\#3 as an adjunctive therapy in relapsing mild-to-moderate ulcerative colitis significantly reduces ulcerative colitis disease activity. Evid Based Med, 2011.

18. Kuisma, J., et al., Effect of Lactobacillus rhamnosus GG on ileal pouch inflammation and microbial flora. Aliment Pharmacol Ther, 2003. 17(4): p. 50915.

19. Nicholls, R.J., et al., Restorative proctocolectomy with ileal reservoir: a pathophysiological assessment. Gut, 1981. 22(6): p. 462-8.

20. Kuisma, J., et al., Factors associated with ileal mucosal morphology and inflammation in patients with ileal pouch-anal anastomosis for ulcerative colitis. Dis Colon Rectum, 2003. 46(11): p. 1476-83.

21. Nasmyth, D.G., et al., Ileal ecology after pouch-anal anastomosis or ileostomy. A study of mucosal morphology, fecal bacteriology, fecal volatile fatty acids, and their interrelationship. Gastroenterology, 1989. 96(3): p. 817-24.

22. Duffy, M., et al., Sulfate-reducing bacteria colonize pouches formed for ulcerative colitis but not for familial adenomatous polyposis. Dis Colon Rectum, 2002. 45(3): p. 384-8.

23. Ohge, H., et al., Association between fecal hydrogen sulfide production and pouchitis. Dis Colon Rectum, 2005. 48(3): p. 469-75.

24. Ruseler-van Embden, J.G., W.R. Schouten, and L.M. van Lieshout, Pouchitis: result of microbial imbalance? Gut, 1994. 35(5): p. 658-64.

25. Komanduri, S., et al., Dysbiosis in pouchitis: evidence of unique microfloral patterns in pouch inflammation. Clin Gastroenterol Hepatol, 2007. 5(3): p. 35260.

26. Iwaya, A., et al., Change in the bacterial flora of pouchitis. HepatoGastroenterology, 2006. 53(67): p. 55-59.

27. Kmiot, W.A., et al., Mucosal morphology, cell proliferation and faecal bacteriology in acute pouchitis. Br J Surg, 1993. 80(11): p. 1445-9. 
28. O'Connell, P.R., et al., Enteric bacteriology, absorption, morphology and emptying after ileal pouch-anal anastomosis. Br J Surg, 1986. 73(11): p. 909-14.

29. McLaughlin, S.D., et al., The bacteriology of pouchitis: a molecular phylogenetic analysis using $16 S$ rRNA gene cloning and sequencing. Ann Surg, 2010. 252(1): p. 90-8.

30. Sandborn, W.J., et al., Pouchitis after ileal pouch-anal anastomosis: a Pouchitis Disease Activity Index. Mayo Clin Proc, 1994. 69(5): p. 409-15.

31. Barton, J.G., et al., Comparison of postoperative outcomes in ulcerative colitis and familial polyposis patients after ileoanal pouch operations. Am J Surg, 2001. 182(6): p. 616-20.

32. Dozois, R.R., et al., Ileal pouch-anal anastomosis: comparison of results in familial adenomatous polyposis and chronic ulcerative colitis. Ann Surg, 1989. 210(3): p. 268-71; discussion 272-3.

33. Zoetendal, E.G., et al., Mucosa-associated bacteria in the human gastrointestinal tract are uniformly distributed along the colon and differ from the community recovered from feces. Appl Environ Microbiol, 2002. 68(7): p. 3401-7.

34. Chou, H.H. and M.H. Holmes, DNA sequence quality trimming and vector removal. Bioinformatics, 2001. 17(12): p. 1093-104.

35. DeSantis, T.Z., et al., Greengenes, a chimera-checked 16S rRNA gene database and workbench compatible with ARB. Appl Environ Microbiol, 2006. 72(7): p. 5069-72.

36. Altschul, S.F., et al., Basic local alignment search tool. J Mol Biol, 1990. 215(3): p. 403-10.

37. Wang, Q., et al., Naive Bayesian classifier for rapid assignment of $r R N A$ sequences into the new bacterial taxonomy. Appl Environ Microbiol, 2007. 73(16): p. 5261-7.

38. Cole, J.R., et al., The Ribosomal Database Project: improved alignments and new tools for rRNA analysis. Nucleic Acids Res, 2009. 37(Database issue): p. D141-5.

39. Lim, M., et al., An assessment of bacterial dysbiosis in pouchitis using terminal restriction fragment length polymorphisms of $16 \mathrm{~S}$ ribosomal DNA from pouch effluent microbiota. Dis Colon Rectum, 2009. 52(8): p. 1492-500.

40. Sekirov, I., et al., Gut microbiota in health and disease. Physiol Rev, 2010. 90(3): p. 859-904.

41. Engelbrektson, A.L., et al., Analysis of treatment effects on the microbial ecology of the human intestine. FEMS Microbiol Ecol, 2006. 57(2): p. 239-50.

42. Shen, B.O., et al., Clostridium difficile infection in patients with ileal pouch-anal anastomosis. Clin Gastroenterol Hepatol, 2008. 6(7): p. 782-8.

43. Lodes, M.J., et al., Bacterial flagellin is a dominant antigen in Crohn disease. J Clin Invest, 2004. 113(9): p. 1296-306.

44. Ferrante, M., et al., Development of pouchitis following ileal pouch-anal anastomosis (IPAA) for ulcerative colitis: A role for serological markers and microbial pattern recognition receptor genes. J Crohns Colitis, 2008. 2(2): p. 142-51.

45. Hirata, N., et al., Proliferation of immature plasma cells in pouchitis mucosa in patients with ulcerative colitis. Inflamm Bowel Dis, 2008. 14(8): p. 1084-90. 
46. Patel, R.T., et al., Cytokine production in pouchitis is similar to that in ulcerative colitis. Dis Colon Rectum, 1995. 38(8): p. 831-7.

47. Stallmach, A., et al., Increased state of activation of CD4 positive T cells and elevated interferon gamma production in pouchitis. Gut, 1998. 43(4): p. 499-505.

48. Sehgal, R., et al., NOD2/CARD15 mutations correlate with severe pouchitis after ileal pouch-anal anastomosis. Dis Colon Rectum, 2010. 53(11): p. 1487-94.

49. Bouskra, D., et al., Lymphoid tissue genesis induced by commensals through NOD1 regulates intestinal homeostasis. Nature, 2008. 456(7221): p. 507-10.

50. Mazmanian, S.K., et al., An immunomodulatory molecule of symbiotic bacteria directs maturation of the host immune system. Cell, 2005. 122(1): p. 107-18.

51. Arumugam, M., et al., Enterotypes of the human gut microbiome. Nature, 2011. 473(7346): p. 174-80. 